\title{
Editorial
}

\section{Herramientas software en el mundo del electromagnetismo computacional}

A partir de los años 80 y durante décadas, las herramientas de simulación empleadas en el mundo electromagnético computacional han tenido una gran demanda, pero hoy en día, gracias a los avances tecnológicos relacionados sobre todo con la computación y los métodos numéricos, ha hecho posible que, con mayor facilidad, se pueda investigar y realizar simulaciones electromagnéticas. Estas herramientas software abarcan un amplio margen de análisis y diseño, como son: el estudio de la compatibilidad electromagnética entre equipos en diferentes entornos, el diseño y análisis de antenas, análisis y diseño de circuitos, componentes pasivos de microondas, cálculo de la sección radar (Radar Cross Section, RCS) y de imágenes ISAR (Inverse Synthetic Aperture Radar), análisis de antenas embarcadas sobre estructuras complejas, análisis Doppler, radio propagación tanto en entornos exteriores como en interiores, análisis de sistemas radio y estudio del acoplo entre antenas, etc.

En el mundo de la ingeniería, de la investigación e incluso de los estudiantes de posgrado, dichas herramientas de simulación de campos electromagnéticos se han vuelto necesarias para la toma de decisiones. Siendo una alternativa importante a la realización de medidas en entornos reales, debido a que es un medio interactivo y dinámico que brinda la oportunidad de analizar escenarios complejos del mundo real de forma precisa, con un bajo coste económico y un ahorro de tiempo considerable. De todos es conocido que para realizar los análisis y diseños anotados anteriormente, existen dos alternativas. Por un lado, realizar medidas usando una estructura real o bien un modelo escalado. Por otro lado, se tiene la alternativa de emplear una herramienta software basada en técnicas numéricas. En el caso de la toma de medidas, considerando tanto la opción de emplear la estructura real, o bien, un prototipo de la misma realizado a escala, en ambos casos se tiene aso- 
ciado un elevado valor económico, debido al alto coste de las instalaciones y el mantenimiento de las mismas donde las medidas son hechas, así como las horas necesarias para llevar a cabo dichas medidas.

Frente a estas desventajas que presentan la realización de medidas, las herramientas software suponen una gran alternativa a la industria e investigación. Debido a que con un menor coste económico y temporal, se pueden construir escenarios y diseños reales para su análisis, permitiendo ajustar las características del diseño y detectar los principales problemas, que de este proceso se deriven, con antelación. Las herramientas software de simulación electromagnética están basadas en dos tipos de técnicas: técnicas rigurosas y técnicas asintóticas. Las técnicas rigurosas, tal como el Método de los Momentos (MoM), Finite Difference Time Domain (FDTD), o el Método de los Elementos Finitos (FEM), todas ellas están basadas en la discretización de la geometría en elementos diferenciales. Por lo que, para estructuras eléctricamente grandes, los recursos computacionales requeridos se incrementan y estas técnicas llegan a ser limitadas por la disponibilidad de recursos computacionales, aunque gracias a las nuevas investigaciones, estas limitaciones cada vez son menores. Pero, a pesar de este inconveniente, se obtiene una solución exacta numéricamente del problema.

Las técnicas asintóticas tienen la principal ventaja de que su coste computacional es independiente de la frecuencia de análisis, sin embargo, la precisión de los resultados incrementa al incrementar la frecuencia. Por lo que estos métodos son especialmente adecuados para analizar problemas eléctricamente grandes, particularmente en el proceso de diseño donde el número de análisis puede ser muy alto. Las técnicas más populares son Óptica Geométrica y la Teoría Uniforme de la Difracción (GO/UTD) y la Física Óptica junto con la Teoría Física de la Difracción (PO/PTD).

La precisión de ambas técnicas está íntimamente relacionada con la fidelidad del modelo geométrico comparado con el escenario real. Esta relación de dependencia directa hace que la exactitud en los resultados obtenidos mejore cuanto más se aproxime el modelo geométrico a la estructura real. Una primera aproximación consiste en simplificar el modelo geométrico empleando estructuras ca- 
nonícas para hacer más sencillo el tratamiento geométrico asociado con las técnicas rigurosas y asintóticas. La principal desventaja de esta opción es una pérdida de la precisión en los resultados. La segunda opción es obtener un modelo exacto del escenario que nos proporcione resultados que sean tan precisos cómo sea posible. En este caso, el precio es el coste computacional asociado con la solución. No obstante, si se combinan los métodos electromagnéticos con alguna técnica de tratamiento geométrico o de aceleración de trazado de rayos, esto nos permite alcanzar la solución de problemas realísticos y complejos con una carga computacional razonable. Por lo que el papel que juega en este tipo de análisis el modelado geométrico de la estructura real, es tan importante como la implementación de las eficientes técnicas numéricas. Siendo esta la principal causa, por la que, se utilizan superficies NURBS (NonUniform Rational B-Spline) para el modelado de las estructuras reales. Estas han sido las superficies paramétricas más populares en el mundo del electromagnetismo computacional ya que se ajustan perfectamente a la estructura real requiriendo almacenar muy poca información para definirla. Además estas superficies representan el enlace perfecto desde el mundo del electromagnetismo a otros mundos. De este modo queda perfectamente justificado el uso de este tipo de superficies en el proceso de modelado de la estructura.

En el ámbito del software de análisis electromagnético, el Grupo de Electromagnetismo Computacional de la Universidad de Alcalá ha desarrollado la herramienta NEWFASANT (www.fasant.com), la cual permite realizar cualquier tipo de análisis y diseño electromagnético mediante la aplicación tanto de técnicas rigurosas como asintóticas. Además posee un potente módulo de tratamiento geométrico que permite modelar estructuras mediante superficies NURBS, así como trabajar con modelos obtenidos a través de otros programas de diseño asistido por ordenador como puede ser AUTOCAD o Rhinoceros.

\section{LORENA LOZANO PLATA}

Universidad de Alcalá

Alcalá de Henares, España 
